\title{
Efficient decoupling schemes with bounded controls based on Eulerian orthogonal arrays
}

\author{
Pawel Wocjan* \\ Institute for Quantum Information, California Institute of Technology M/C 107-81, Pasadena, California 91125, USA
}

(Received 6 March 2006; published 13 June 2006)

\begin{abstract}
The task of decoupling, i.e., removing unwanted internal couplings of a quantum system and its couplings to an environment, plays an important role in quantum control theory. There are many efficient decoupling schemes based on combinatorial concepts such as orthogonal arrays, difference schemes, and Hadamard matrices. So far these combinatorial decoupling schemes have relied on the ability to effect sequences of instantaneous, arbitrarily strong control Hamiltonians (bang-bang controls). To overcome the shortcomings of bang-bang control, Viola and Knill proposed a method called "Eulerian decoupling" that allows the use of bounded-strength controls for decoupling. However, their method was not directly designed to take advantage of the local structure of internal couplings and couplings to an environment that typically occur in multipartite quantum systems. In this paper we define a combinatorial structure called Eulerian orthogonal array. It merges the desirable properties of orthogonal arrays and Eulerian cycles in Cayley graphs (that are the basis of Eulerian decoupling). We show that this structure gives rise to decoupling schemes with bounded-strength control Hamiltonians that can be used to remove both internal couplings and couplings to an environment of a multipartite quantum system. Furthermore, we show how to construct Eulerian orthogonal arrays having good parameters in order to obtain efficient decoupling schemes.
\end{abstract}

DOI: 10.1103/PhysRevA.73.062317

PACS number(s): 03.67.Lx, 03.65.Fd

\section{INTRODUCTION}

An important task in quantum control theory consists in selectively removing unwanted contributions of the system Hamiltonian and/or switching off couplings of the quantum system to an uncontrollable environment (the latter being responsible for decoherence). This task is usually called decoupling (see, e.g., Refs. [1-4] and, e.g., Refs. [5-9] for schemes using combinatorial concepts). More generally, one is also interested in effectively changing the system Hamiltonian in order to simulate some desired Hamiltonian; this is usually referred to as simulating Hamiltonians (see, e.g., Refs. [9-12]). In this paper we concentrate on designing efficient decoupling schemes.

Methods of dynamical decoupling and also simulating Hamiltonians derive their basic physical intuition from coherent averaging techniques in high-resolution NMR spectroscopy $[13,14]$. A decoupling scheme is understood as a control protocol which relies on the repeated application of controls drawn from a finite set in order to change effectively the natural time evolution to the desired evolution. Many efficient decoupling schemes can be designed with the help of combinatorial concepts such as Hadamard matrices, difference schemes, and orthogonal arrays. The entries of these structures describe how to choose the controls. The reason why it is possible to use these combinatorial objects is the local structure of the system Hamiltonians (pair interactions or, more generally, few-body Hamiltonians) and the couplings to the environment. All these combinatorial schemes rely on the ability to effect sequences of instantaneous, arbitrarily strong control Hamiltonians (bang-bang controls). Such schemes are unrealistic in many situations. To

\footnotetext{
*Electronic address: wocjan@cs.caltech.edu
}

overcome the shortcomings of bang-bang decoupling, Viola and Knill proposed a general method called Eulerian decoupling for implementing decoupling with bounded controls, i.e., continuously modulated bounded-strength control Hamiltonians $[15,16]$. This method offers many advantages over bang-bang decoupling. However, their method was not directly designed to make use of the local structure of internal couplings and couplings to an environment in order to reduce the complexity of decoupling.

We show how to incorporate some of the abovementioned combinatorial methods (used, so far, only in the bang-bang formulation) into the method of Viola and Knill in order to obtain efficient decoupling schemes with bounded controls. Our decoupling schemes can be applied to multipartite quantum systems to remove both internal couplings and couplings to an environment. They rely on a combinatorial object which we call Eulerian orthogonal array. We show how to construct these objects with good parameters from error correcting codes.

The paper is organized as follows. In Sec. II we describe the principles of dynamical decoupling. We describe briefly the so-called first-order approximation that is the basis for all decoupling schemes. In Sec. III we recall how to construct decoupling schemes with bang-bang and bounded-strength controls. The method using bounded-strength control is Viola and Knill's Eulerian decoupling, which assumes no special structure of the quantum system. In Sec. IV we consider quantum systems consisting of coupled qudits. Section IV A reviews how to construct efficient decoupling schemes with bang-bang controls and orthogonal arrays. In Sec. IV B we show how to obtain efficient decoupling schemes with bounded controls, using Eulerian orthogonal arrays which merge the concept of orthogonal arrays with the idea of Eulerian decoupling. In Sec. V we provide constructions of Eulerian orthogonal arrays with good parameters. 


\section{PRINCIPLES OF DYNAMICAL DECOUPLING}

A decoupling scheme is a control protocol which effectively changes the natural time evolution to the desired evolution by repeated application of controls drawn from a finite set. We refer the reader to Refs. $[15,16]$ for a more detailed description. In the following we give a brief introduction based on the above articles.

The joint evolution of the target system $S$ and the environment $E$ is described by a total drift Hamiltonian of the form

$$
H=H_{S} \otimes \mathbf{1}_{E}+\mathbf{1}_{S} \otimes H_{E}+H_{S E}, \quad H_{S E}=\sum_{a} S_{a} \otimes E_{a},
$$

where $H_{S}$ and $H_{E}$ characterize the isolated dynamics of the system and the environment, respectively, and the interaction term $H_{S E}$ is responsible for introducing unwanted decoherence effects and dissipation effects in the reduced dynamics of $S$ alone. Without loss of generality we always choose the operators $S_{a}$ and $H_{S}$ to be traceless.

The idea behind dynamical decoupling is to add a specially designed controller, described by a time-dependent control Hamiltonian $H_{c}(t)$ acting on only the target system $S$, in such a way that the resulting controlled dynamics is described by an effective Hamiltonian $H_{\text {eff }}$ which no longer contains any coupling terms between $S$ and $E$, i.e.,

$$
H_{\mathrm{eff}}=\widetilde{H}_{S} \otimes \mathbf{1}_{E}+\mathbf{1}_{S} \otimes H_{E},
$$

for an appropriate, possibly modified, system Hamiltonian $\widetilde{H}_{S}$. In this paper we are interested in the case that $\widetilde{H}_{S}=\mathbf{0}$.

Decoupling protocols are most conveniently constructed by directly looking at the control propagator associated to $H_{c}(t)$,

$$
U_{c}(t)=\mathcal{T} \exp \left\{-i \int_{0}^{t} H_{c}(\tau) d \tau\right\},
$$

where $\mathcal{T}$ denotes the time ordering.

The control actions are always cyclic, i.e., $U_{c}\left(t+T_{c}\right)$ $=U_{c}(t)$ for some cycle time $T_{c}$ and for all $t$. The stroboscopic dynamics $U\left(t_{M}\right)$ with $t_{M}=M T_{c}$ and $M \in \mathbb{N}$ may be described by a propagator

$$
U\left(t_{M}\right)=\exp \left(-i \bar{H} t_{M}\right)
$$

for a time-independent effective Hamiltonian $H_{\text {eff. }}$ If, in addition, $T_{c}$ is sufficiently short, then the effective Hamiltonian is accurately represented by the following lowest-order Hamiltonian:

$$
\bar{H}=\frac{1}{T_{c}} \int_{0}^{T_{c}} U_{c}^{\dagger}(t) H U_{c}(t) d \tau
$$

While higher-order terms can be systematically evaluated, the approximation in (5) becomes more and more exact as the fast control limit $T_{c} \rightarrow 0$ is approached. Throughout the paper we consider first-order decoupling which is based on the approximation in Eq. (5).

\section{DECOUPLING SCHEMES}

In this section we do not assume any special structure of the target system $S$, the Hamiltonian $H_{S}$, and the coupling to the environment given by $S_{a}$ 's. We first discuss how to realize decoupling with controls of unbounded strength (bangbang) and then with bounded control (Eulerian decoupling). The presentation is based on Ref. [16].

\section{A. Bang-bang control}

The time average in (5) can be expressed directly as an average over a group in the following simple bang-bang decoupling setting. Let $G$ be a discrete group of order $|G|$ acting on the Hilbert space of the target system $\mathcal{H}_{S}$ via a faithful, unitary, projective representation,

$$
\rho:\left\{\begin{array}{l}
G \rightarrow \mathcal{U}\left(\mathcal{H}_{S}\right) \\
g \mapsto U_{g}:=\rho(g),
\end{array}\right.
$$

where $\mathcal{U}\left(\mathcal{H}_{S}\right)$ denotes the group of unitary matrices acting on $\mathcal{H}_{S}$. Let $\lambda \in \mathbb{N}$ and $\lambda>0$.

A decoupling scheme using $U_{g}$ 's as control operations is specified by a sequence $\left(g_{1}, g_{2}, \ldots, g_{N}\right)$ with $N:=\lambda|G|$ and entries from $G$. The number $N$ is called the length of the decoupling scheme. The entries $g_{j}$ 's specify the control propagator $U_{c}(t)$ over each of the $\lambda|G|$ equally long subintervals. A control cycle is defined by

$$
U_{c}((j-1) \Delta+\tau)=U_{g_{j}}, \quad \tau \in[0, \Delta),
$$

with $T_{c}=\lambda|G| \Delta$ for some $\Delta>0$, and $j=1, \ldots, N$.

If all group elements appear exactly $\lambda$ times in the list $\left(g_{1}, \ldots, g_{N}\right)$ then the resulting control action corresponds to extracting the $G$-invariant component of $X$. We have

$$
\frac{1}{N} \sum_{j=1}^{N} U_{g_{j}}^{\dagger} X U_{g_{j}}=\frac{\lambda}{N} \sum_{g \in G} U_{g}^{\dagger} X U_{g}=\Pi_{G}(X),
$$

where

$$
\Pi_{G}(X)=\frac{1}{|G|} \sum_{g \in G} U_{g}^{\dagger} X U_{g} .
$$

Note that if the representation $\rho$ in (6) is irreducible then we have $\Pi_{G}(X)=\operatorname{tr}(X) / d \mathbf{1}_{d}$ for all $X$, where $d$ is the dimension of $\mathcal{H}_{S}$.

An example for such an irreducible, unitary, projective representation is given in the following. The discrete Fourier transform of length $d \in \mathbb{N}$ is the unitary transformation defined by $\mathrm{DFT}_{d}:=\frac{1}{\sqrt{d}} \sum_{k, \ell=0}^{d-1} \omega^{k \cdot \ell}|k\rangle\langle\ell|$, where $\omega$ denotes the primitive $d$ th root of unity $e^{2 \pi i / d}$. Next, define operators $S:=\sum_{k=0}^{d-1}|k\rangle\langle k+1|$, where the indices are reduced modulo $d$, and $T:=\mathrm{DFT}_{d}^{\dagger} \cdot S \cdot \mathrm{DFT}_{d}=\sum_{k=0}^{d-1} \omega^{k}|k\rangle\langle k|$. Then the map

$$
\rho:\left\{\begin{array}{l}
Z_{d} \times Z_{d} \rightarrow \mathcal{U}(d) \\
(a, b) \mapsto S^{a} T^{b}
\end{array}\right.
$$


is an irreducible, unitary, projective representation. Note that for $d=2$ one obtains $\mathbf{1}, \sigma_{x}, \sigma_{y}, \sigma_{z}$, where the $\sigma$ 's are the Pauli matrices.

Now it is clear that we can remove the couplings to the environment and switch off the natural time evolution of the quantum system by performing the control operations according to (7) and the representation in (9). This is because $\Pi_{G}\left(S_{a}\right)=\mathbf{0}$ for all $a$ and $\Pi_{G}\left(H_{S}\right)=\mathbf{0}$. However, this method has the following disadvantage that makes it unrealistic for many situations. According to the rule in (7) the control propagator $U_{c}(t)$ jumps from $U_{g_{j-1}}$ to $U_{g_{j}}=\left(U_{g_{j}} U_{g_{j-1}}^{\dagger}\right) U_{g_{j}}$ through the application of an arbitrarily strong, instantaneous kick at the $j$ th endpoint $t_{j}=j \Delta$, realizing the bang-bang pulse $U_{s_{j}}=U_{g_{j}} U_{g_{j-1}}^{\dagger}$ with $s_{j}:=g_{j} g_{j-1}^{-1}$ (equality is understood here up to a phase factor). In the next section we describe how to avoid such bang-bang controls.

\section{B. Eulerian decoupling}

As already mentioned in the Introduction the requirements for bang-bang control are highly unrealistic. Viola and Knill proposed a method called Eulerian decoupling [16] that avoids the use of such bang-bang pulses. In Eulerian decoupling the control propagator $U_{c}(t)$ is varied smoothly from $U_{g_{j-1}}$ to $U_{g_{j}}$ by a control action distributed along the whole $j$ th subinterval.

Let $S$ be a generating set for $G$, i.e., any element of $G$ can be written as a product of elements of $S$. The Cayley graph $\Gamma(G, S)$ of $G$ with respect to $S$ is a directed graph whose vertices are labeled by the group elements and whose edges are labeled by the generators. More precisely, the vertex $g$ is joined to the vertex $h$ if and only if $g h^{-1}=s$ for some $s \in S$, i.e., $g=s h$.

It is assumed that we have the ability to physically implement the generators $s \in S$, i.e., to implement the unitaries $U_{s}$ by the application of some suitably chosen control Hamiltonians $h_{s}(t)$ over $\Delta$ :

$$
U_{s}=u_{s}(\Delta)
$$

where

$$
u_{s}(\delta)=\mathcal{T}\left\{\exp \left[-i \int_{0}^{\delta} h_{s}(\tau) d \tau\right]\right\}
$$

for $\delta \in[0, \Delta]$. The choice of the control Hamiltonians $h_{s}(t)$ is not unique. This allows for additional flexibility for the concrete implementation. Once a choice of the control Hamiltonians is made, the control action is determined by assigning a cycle time and a rule for switching the Hamiltonians $h_{s}(t)$ during the cycle subintervals.

Viola and Knill [16] showed that decoupling can be achieved by sequentially implementing generators so that they follow a Eulerian cycle in $\Gamma(G, S)$. An Eulerian cycle is defined as a cycle that uses each edge exactly once. Because a Cayley graph is regular, it always has an Eulerian cycle, whose length is necessarily $N=|G||S|$ (see, e.g., Refs. [17,18] for the definition of these notions). For our purposes, we use a slightly more general definition: an Eulerian cycle with multiplicity $\lambda$ is a cycle that uses each edge exactly $\lambda$ times. Clearly, such an Eulerian cycle necessarily has length $N=\lambda|G||S|$. We choose an Eulerian cycle to begin at the identity element of $G$. Therefore, an Eulerian cycle can be described as a list $\left(s_{1}, \ldots, s_{N}\right)$ with entries from $S$. Each entry identifies the edge via which we leave the vertex.

Decoupling according to an Eulerian cycle $\mathcal{C}:=\left(s_{1}, \ldots, s_{N}\right)$ is defined by setting the cycle time $T_{c}=N \Delta$ and by choosing the control propagators $U_{c}(t)$ as follows:

$$
U_{c}[(j-1) \Delta+\delta]=u_{s_{j}}(\delta) U_{c}[(j-1) \Delta]
$$

where $\delta \in[0, \Delta)$ and $u_{s_{j}}(\delta)$ is defined in (10) and (11). This decoupling prescription means that during the $j$ th subinterval one chooses as a control Hamiltonian the one that realizes the generator $s_{j}$, i.e., the $j$ th element of $\mathcal{C}$.

The effective Hamiltonian $\bar{H}$ under Eulerian decoupling is obtained by evaluating the time average in (5) with the control propagator being given by (12). The resulting $N$ terms can be partitioned into $|S|$ families, each corresponding to a fixed generator. Because for each $s$ the cycle $\mathcal{C}$ contains exactly $\lambda s$-labeled edges ending at any given vertex $g$, each family leads to a sum over the group elements as in (8).

For these reasons the quantum operation $\mathcal{Q}_{\mathcal{C}}$ defined by $\mathcal{C}$ can be decomposed as

$$
\mathcal{Q}_{\mathcal{C}}(X)=\Pi_{G}\left[F_{S}(X)\right]
$$

with the map $F_{S}$ implementing an average over both the group generators and control subinterval

$$
F_{S}(X)=\frac{1}{|S|} \sum_{s \in S} \frac{1}{\Delta} \int_{0}^{\Delta} u_{s}(\tau)^{\dagger}(s) X u_{s}(\tau) d \tau .
$$

The link between Eulerian decoupling and bang-bang decoupling by averaging over $G$ is established in the following theorem. Some additional compatibility between $\Pi_{G}$ and $F_{S}$ is necessary [16]. Let us repeat all the notions before stating the theorem. Let $G$ be a group that acts via a faithful, unitary, projective representation $g \mapsto U_{g}$ on $\mathbb{C}^{d}$. The decoupling group algebra $\mathcal{D}$ of $G$ is the C-linear span of the matrices $U_{g}$.

Theorem 1 (Eulerian decoupling). Let $X$ be any operator acting on $\mathrm{C}^{d}$. If the control Hamiltonians $h_{s}(t)$ are in the decoupling group algebra, i.e., $h_{s}(\delta) \in \mathcal{D}$ for all times $\delta \in[0, \Delta]$ and all $s \in S$, then Eulerian decoupling according an Eulerian cycle $\mathcal{C}$ as specified by the rule in (12) has the same effect as averaging over $G$ as in (8), i.e.,

$$
\mathcal{Q}_{\mathcal{C}}(X)=\Pi_{G}(X) .
$$

For the proof we refer the reader to Ref. [16]. Note that the bang-bang limit is formally recovered by substituting the map $F_{S}$ by the identity map. In the Eulerian approach, at the expense of lengthening the control cycle, the same $G$ symmetrization can be attained using only bounded-strength controls. The maximum strength achievable in implementing the generators directly bounds the minimum attainable $T_{c}$, and therefore the accuracy of the first-order approximation. 


\section{EFFICIENT DECOUPLING SYSTEMS}

In this section we consider a target system that is composed of $n$ coupled qudits, i.e., its Hilbert space $\mathcal{H}_{S}$ is given by the tensor product $\mathcal{H}_{S}=\left(\mathbb{C}^{d}\right)^{\otimes n}$. We say that a family of decoupling schemes is efficient if the number of control operations grows polynomially with the number of qudits. So far there were only efficient decoupling schemes using bangbang controls (see, e.g., the references given in Sec. I). The schemes rely on the special structure of the system Hamiltonians and the couplings to the environment. It is assumed that the system Hamiltonian is a so-called few-body Hamiltonian. To define this precisely we need to introduce some notions. For any operator $A$ acting on $C^{d}$ we denote by $A^{(k)}$ the operator that acts as $A$ on the $k$ th qudit, i.e., $A^{(k)}=\mathbf{1}$ $\otimes \cdots \otimes \mathbf{1} \otimes A \otimes \mathbf{1} \otimes \cdots \otimes \mathbf{1}$

Let $\mathcal{B}:=\left\{\sigma_{\alpha} \mid \alpha=1, \ldots, d^{2}\right\}$ be a basis of for the vector space $\mathrm{C}^{d \times d}$ of matrices acting on $\mathrm{C}^{d}$. We say that an operator $X$ acts on the qudits $k_{1}, \ldots, k_{t}$ with $1 \leqslant k_{1}<\cdots<k_{t} \leqslant n$ if it can be expressed as follows:

$$
X=\sum_{\alpha_{1}, \ldots, \alpha_{t}} x_{\alpha_{1}, \ldots \alpha_{t}} \sigma_{\alpha}^{\left(k_{1}\right)} \cdots \sigma_{\beta}^{\left(k_{t}\right)}
$$

for some $x_{\alpha_{1}, \ldots, \alpha_{t}} \in \mathrm{C}$.

We assume that the system Hamiltonian is a $t$-body Hamiltonian, i.e., it can be decomposed as

$$
H_{S}:=\sum_{k_{1}, \ldots, k_{t}} H_{k_{1}, \ldots, k_{t}}
$$

where $H_{k_{1}, \ldots, k_{t}}$ are traceless operators acting on qudits $k_{1}, \ldots, k_{t}$ only. For $t=2$ one also says that $H$ is a pair interaction Hamiltonian. Furthermore, we assume that the environment couples to $t$-tuples of qudits, i.e., we have

$$
H_{S E}=\sum_{k_{1}, \ldots, k_{t}} S_{k_{1}, \ldots, k_{t}} \otimes E_{k_{1}, \ldots, k_{t}},
$$

where $S_{k_{1}, \ldots, k_{t}}$ are traceless operators acting on qubits $k_{1}, \ldots, k_{t}$ only and $E_{k_{1}, \ldots, k_{t}}$ act on the Hilbert space $\mathcal{H}_{E}$ of the environment.

It is convenient to use the following definition. Let $X$ be an arbitrary operator acting on $\left(\mathbb{C}^{d}\right)^{\otimes t}$. We define its embed$\operatorname{ding} X^{\left(k_{1}, \ldots, k_{t}\right)}$ into $\left(\mathbb{C}^{d}\right)^{\otimes n}$ to be the operator

$$
X^{\left(k_{1}, \ldots, k_{t}\right)}=\sum_{\alpha_{1}, \ldots, \alpha_{t}} x_{\alpha_{1}, \ldots \alpha_{t}} \sigma_{\alpha_{1}}^{\left(k_{1}\right)} \cdots \sigma_{\alpha_{t}}^{\left(k_{t}\right)},
$$

where $X=\Sigma_{\alpha_{1}, \ldots, \alpha_{t}} x_{\alpha_{1}, \ldots \alpha_{t}} \sigma_{\alpha_{1}} \otimes \cdots \otimes \sigma_{\alpha_{t}}$ is the expansion of $X$ in the product basis $\mathcal{B}^{\otimes t}$.

\section{A. Decoupling with bang-bang controls based on orthogonal arrays}

We assume that we can perform bang-bang controls on each qudit individually. Formally, all control operations are elements of some finite subset of the group $\mathcal{U}(d)^{\otimes n}$, where $\mathcal{U}(d)$ denotes the group of unitary matrices acting on $\mathrm{C}^{d}$. In the following we recall how orthogonal arrays (OAs) may be used to construct efficient decoupling schemes. Orthogonal arrays appeared first in statistics where they were used in the design of experiments for collecting statistical data systematically. We refer the reader to the books [19-21] for applications and constructions of orthogonal arrays. Stollsteimer and Mahler first used orthogonal arrays for the construction of decoupling schemes and selective coupling schemes [8] for qubit systems with pair interactions. This method was generalized to qudit systems with $t$-local interactions in $[9,22]$.

Definition 1 (orthogonal array of strength $t$ ). Let $\mathcal{A}$ be a finite alphabet and let $n, N \in \mathbb{N}$. An $n \times N$ array $M$ with entries from $\mathcal{A}$ is an orthogonal array with $s=|\mathcal{A}|$ levels, strength $t$, and multiplicity $\lambda$ if and only if every $t \times N$ subarray of $M$ contains each possible $t$ tuple of elements in $\mathcal{A}^{t}$ precisely $\lambda$ times as a column. We use the notation $\mathrm{OA}_{\lambda}(N, n, s, t)$ to denote a corresponding orthogonal array. If $\lambda, s$, and $t$ are understood we also use the shorthand notation $\mathrm{OA}(N, n)$.

An important special case arises if the strength $t$ is 2 . This means that each pair of elements of $\mathcal{A}$ occurs $\lambda$ times in the list $\left[\left(a_{k j}, a_{l j}\right) \mid j=1, \ldots N\right]$ for $1 \leqslant k<l \leqslant n$. Most of the known construction actually yield arrays of strength two [21]. For many physical systems it is sufficient to study arrays of small strength since the strength relates to the degree of the interactions, i.e., for pair interaction Hamiltonians it is sufficient to consider arrays of strength $t=2$. For an example of such orthogonal arrays see Refs. [22,23].

The basic idea is to use an orthogonal array $M$ with parameters $\mathrm{OA}\left(N, n, d^{2}, 2\right)$ over an alphabet $\mathcal{A}$ of size $d^{2}$. Here $d$ denotes the dimension of the qudits. The elements of $\mathcal{A}$ are identified with the elements of the group $G:=Z_{d} \times Z_{d}$ that acts irreducibly on $\mathbb{C}^{d}$ via the map in (9). The columns $\left(g_{1 j}, \ldots, g_{n j}\right)^{T}$ of $M$ specify the control propagators $U_{c}(t)$ over each of the $N$ equally long subintervals. A control cycle is defined by

$$
U_{c}[(j-1) \Delta+\tau]=U_{g_{1 j}} \otimes U_{g_{2 j}} \otimes \cdots \otimes U_{g_{n j}},
$$

where $\tau \in[0, \Delta), T_{c}=N \Delta$ for some $\Delta>0$, and $j=1, \ldots, N$.

The following theorem shows that the prescription in (18) allows us to decouple few-body Hamiltonians and couplings to the environment. We denote by $G^{\times t}$ the direct product group $G \times \cdots \times G$ having $t$ components.

Theorem 2 (decoupling with OAs). Let $G:=Z_{d} \times Z_{d}$ and $g \mapsto U_{g}$ be the irreducible, unitary, projective representation as in (9). Let $M=\left(g_{k j}\right)$ be an $\mathrm{OA}(n, N)$ be an orthogonal array of strength $t$ over the group $G$. Let $\Pi_{M}$ denote the control action that corresponds to (18). Then we have

$$
\Pi_{M}\left(X^{\left(k_{1}, \ldots, k_{t}\right)}\right)=\mathbf{0}
$$

for an arbitrary traceless operator acting on $\left(\mathbb{C}^{d}\right)^{\otimes t}$ and any $t$ tuple $\left(k_{1}, \ldots, k_{t}\right)$ with $1 \leqslant k_{1}<\cdots<k_{t} \leqslant n$.

Proof. The idea is to reduce the problem for each $t$ tuple $\left(k_{1}, \ldots, k_{t}\right)$ to the case in $(8)$ by using the special structure of the operator $X^{\left(k_{1}, \ldots, k_{t}\right)}$. We have 


$$
\begin{aligned}
\Pi_{M}\left(X^{\left(k_{1}, \ldots, k_{t}\right)}\right) & =\frac{1}{N} \sum_{j=1}^{N}\left(U_{g_{1 j}} \otimes U_{g_{2 j}} \otimes \cdots \otimes U_{g_{n j}}\right)^{\dagger} X^{\left(k_{1}, \ldots, k_{t}\right)}\left(U_{g_{1 j}} \otimes U_{g_{2 j}} \otimes \cdots \otimes U_{g_{n j}}\right) \\
& =\left[\frac{1}{N} \sum_{j=1}^{N}\left(U_{g_{k_{1}, j}} \otimes U_{g_{k_{2}, j}} \otimes \cdots \otimes U_{\left.g_{k_{t}}\right)^{\dagger}} \otimes\left(U_{g_{k_{1}, j}} \otimes U_{g_{k_{2}, j}} \otimes \cdots \otimes U_{g_{k_{r} j}}\right)\right]^{\left(k_{1}, \ldots, k_{t}\right)}\right. \\
& =\left[\frac{\lambda}{N} \sum_{\left(h_{1}, \ldots, h_{t}\right) \in G^{\times t}}\left(U_{h_{1}} \otimes U_{h_{2}} \otimes \cdots \otimes U_{h_{t}}\right)^{\dagger} X\left(U_{h_{1}} \otimes U_{h_{2}} \otimes \cdots \otimes U_{\left.h_{t}\right)}\right)\right]^{\left(k_{1}, \ldots, k_{t}\right)} \\
& =\left[\Pi_{G^{\times t}}(X)\right]^{\left(k_{1}, \ldots, k_{t}\right)}=\mathbf{0}^{\left(k_{1}, \ldots, k_{t}\right)}=\mathbf{0} .
\end{aligned}
$$

The first equality is because $X^{\left(k_{1}, \ldots, k_{t}\right)}$ acts on the qudits $k_{1}, \ldots, k_{t}$ only. Note that the representation of $G^{\times t}$ to $\mathcal{U}(d)^{\otimes t}$ given by $\left(h_{1}, \ldots, h_{t}\right) \mapsto U_{h_{1}} \otimes \cdots \otimes U_{h_{t}}$ is irreducible. Since $M$ is an orthogonal array of strength $t$ the list $\left[\left(g_{k_{1}, j}, \ldots, g_{k_{t}, j}\right)\right]_{j=1}^{N}$ contains every element of $G^{\times t}$ exactly $\lambda$ times. Therefore, we average over the group $G^{\times t}$ acting via an irreducible representation as in (8). This proves Eq. (20).

It is clear that we can switch off all Hamiltonians of the form in (15) and all couplings to an environment of the form in (16) with control actions specified by the above theorem. This is because all operators $H_{k_{1}, \ldots, k_{t}}$ and $S_{k_{1}, \ldots, k_{t}}$ have the form $X^{\left(k_{1}, \ldots, k_{t}\right)}$ for some traceless operator $X$ acting on $\left(C^{d}\right)^{\otimes t}$.

\section{B. Decoupling with bounded-strength controls based on Eulerian orthogonal arrays}

Finally, we show how to combine the ideas of Eulerian decoupling and orthogonal arrays. This is done by introducing the concept of Eulerian orthogonal arrays.

Definition 2 (Eulerian orthogonal array). A $n \times N$-matrix $M=\left(g_{k j}\right)$ with entries from the group $G$ is said to be an Eulerian orthogonal array of strength $t$ iff for all $t$ tuples $\left(k_{1}, k_{2}, \ldots, k_{t}\right)$ with $1 \leqslant k_{1}<\cdot<k_{t} \leqslant n$ there is a generating set $S_{k_{1}, k_{2}, \ldots, k_{t}}$ of $G^{\times t}$ such that the list of group elements

$$
\left(g_{k_{1}, j}, g_{k_{2}, j}, \ldots, g_{k_{t}, j}\right)_{j=1}^{N}
$$

defines an Eulerian cycle in the Cayley graph $\Gamma\left(G^{\times t}, S_{k_{1}, k_{2}, \ldots, k_{t}}\right)$.

Note that the above conditions automatically imply that $M$ is a (usual) orthogonal array of strength $t$.

We assume that we have the ability to implement the group elements $g \in G$, i.e., to implement the unitaries $U_{g}$ on the individual qudits by the application of control Hamiltonians $h_{g}(t)$ over $\Delta$ as in (10) and (11). This means that we have the ability to switch on the control Hamiltonians $h_{g}(t)$ on any qudit, i.e., $\mathbf{1} \otimes \cdots \otimes \mathbf{1} \otimes h_{g}(t) \otimes \mathbf{1} \otimes \cdots \otimes \mathbf{1}$.

We define decoupling according to an Eulerian orthogonal array $M=\left(g_{k j}\right)$ by setting the cycle time $T_{c}=N \Delta$ and by assigning the control propagators as follows:

$$
U_{c}[(j-1) \Delta+\delta]=\left[u_{s_{1 j}}(\delta) \otimes \cdots \otimes u_{s_{n j}}(\delta)\right] U_{c}[(j-1) \Delta],
$$

where $\delta \in[0, \Delta)$ and $s_{k j}=g_{k j}^{-1} g_{k, j+1}$ for $j=1, \ldots, N-1$ and $s_{k N}=g_{k N}^{-1} g_{k 1}$. The tuples $\left(s_{k_{1}, j}, \ldots, s_{k_{t}, j}\right)$ are the edges in the Eulerian cycle defined by the rows $k_{1}, \ldots, k_{t}$ of $M$.

Theorem 3 (decoupling with Eulerian OAs). Let $G:=Z_{d}$ $\times Z_{d}$ and $g \mapsto U_{g}$ be the irreducible, unitary, projective representation in (9). Let $M=\left(g_{k j}\right)$ be an Eulerian orthogonal array over $G$ of size $n \times N$ and strength $t$. Let $\mathcal{Q}_{M}$ denote the control action that results from the control propagator defined in (22). Then we have

$$
\mathcal{Q}_{M}\left[X^{\left(k_{1}, \ldots, k_{t}\right)}\right]=\mathbf{0}
$$

for an arbitrary traceless operator $X$ acting on $\left(\mathrm{C}^{d}\right)^{\otimes t}$ and any $t$ tuple $\left(k_{1}, \ldots, k_{t}\right)$ with $1 \leqslant k_{1}<\ldots<k_{t} \leqslant n$.

Proof. Again the idea is to reduce the problem for each $t$ tuple $\left(k_{1}, \ldots, k_{t}\right)$ to the case of Theorem 1 by using the special structure of the operator $X^{\left(k_{1}, \ldots, k_{t}\right)}$. Let us denote by $\mathcal{C}_{k_{1}, \ldots, k_{t}}$ the Eulerian cycle in the Cayley graph $\Gamma\left(G^{\times t}, S_{k_{1}, \ldots, k_{t}}\right)$ that is defined by the rows $k_{1}, \ldots, k_{t}$ of $M$. Then we have

$$
\begin{aligned}
\mathcal{Q}_{M}\left(X^{\left(k_{1}, \ldots, k_{t}\right)}\right) & =\left[\mathcal{Q}_{\mathcal{C}_{k_{1}, \ldots, k_{t}}}(X)\right]^{\left(k_{1}, \ldots, k_{t}\right)} \\
& =\left\{\Pi_{G^{\times t}}\left[F_{S_{k_{1}, \ldots, k_{t}}}(X)\right]\right\}^{\left(k_{1}, \ldots, k_{t}\right)}=\mathbf{0}^{\left(k_{1}, \ldots, k_{t}\right)}=\mathbf{0} .
\end{aligned}
$$

Equation (24) is due to the fact that $X^{\left(k_{1}, \ldots, k_{t}\right)}$ acts on qudits $k_{1}, \ldots, k_{t}$ only. The remaining equalities follow from Theorem 1 because all its conditions are satisfied.

Again it is clear that we can switch off all Hamiltonians of the form in (15) and all couplings to an environment of the form in (16) with control actions specified by the above theorem.

\section{EULERIAN OAS FROM LINEAR ERROR CORRECTING CODES}

We construct Eulerian orthogonal arrays using linear error correcting codes. Let us briefly repeat some basis facts about 
linear error correcting codes and their relationship to orthogonal arrays.

A linear code over the finite field $\mathbb{F}_{q}$ is a $k$-dimensional subspace of the vector space $\mathbb{F}_{q}^{n}$. We consider finite fields of $q=d^{2}$ only. In this case the additive group of the finite field $\mathbb{F}_{q}$ is isomorphic to $Z_{d} \times Z_{d}$; we again use the irreducible representation in (9). The space $\mathbb{F}_{q}^{n}$ is endowed with a metric called Hamming distance. It is defined as follows: for $x=\left(x_{1}, \ldots, x_{n}\right) \in \mathbb{F}_{q}^{n}$ we have that $\operatorname{wt}(x):=\mid\left\{i \in\{1, \ldots, n\}: x_{i}\right.$ $\neq 0\} \mid$. The minimum distance of a linear code $C$ is defined by $d=d_{\min }:=\min \{\operatorname{wt}(c): c \in C, c \neq \mathbf{o}\}$, where $\mathbf{o}$ denotes the zero vector. In this situation we say shortly that $C$ is an $[n, k, d]_{q}$ code. We need the fact that a $[n, k]_{q}$ linear code can be described by a generator matrix $G$ of size $n \times k$ with entries from $\mathbb{F}_{q}$. The matrix $G$ defines the embedding from $\mathbb{F}_{q}^{k}$ to $\mathbb{F}_{q}^{n}$; the code words $c \in C$ are the images of the vectors $m \in \mathbb{F}_{q}^{k}$, i.e., $c=G m$. We need one more definition which is the dual code $C^{\perp}$ of $C$ defined by $C^{\perp}:=\left\{x \in \mathbb{F}_{q}^{n}: x y=0\right.$ for all $\left.y \in C\right\}$; the dot product $x \cdot y$ is given by $\sum_{i=1}^{n} x_{i} y_{i}$. In the following we refer to the minimum distance $d^{\perp}$ of the dual code as the dual distance.

The following theorem ([21], Theorem 4.6) establishes a close relationship between orthogonal arrays and errorcorrecting codes. This theorem was also used in Refs. $[22,23]$.

Theorem 4 (OAs from linear codes). Let $C$ be a linear $[n, k, d]_{q}$ code over $\mathbb{F}_{q}$ with dual distance $d^{\perp}$. Arrange the codewords of $C$ into the columns of a matrix $A \in \mathbb{F}_{q}^{n \times q^{k}}$. Then $A$ is an $\mathrm{OA}\left(q^{k}, n, q, d^{\perp}-1\right)$.

Now we show how to modify the above construction in order to obtain Eulerian orthogonal arrays.

Theorem 5 (Eulerian OAs from linear codes). Let $C$ be a $[n, k]_{q}$ code with dual distance $d^{\perp}$ and $G$ be a generator matrix for $C$. Let $\mathcal{C}:=\left(m_{0}, \ldots, m_{N-1}\right)$ be an Eulerian cycle in the Cayley graph $\Gamma(V, S)$ with multiplicity 1 , where the group is $V:=\mathbb{F}_{q}^{k}$ and the generating set is the group itself, i.e., $S:=\mathbb{F}_{q}^{k}$. The length of such an Eulerian cycle is necessarily $N=q^{2 k}$. Set $t:=d^{\perp}-1$. Then the $n \times N$ matrix $M$ whose columns are defined to be $G m_{j}$ for $j=0, \ldots, N-1$ is an Eulerian orthogonal array over $\mathbb{F}_{q}$ of strength $t$. Furthermore, we have $S_{k_{1}, \ldots, k_{t}}=G^{\times t}$ for all $t$ tuples $\left(k_{1}, \ldots, k_{t}\right)$ with $1 \leqslant k_{1}<\ldots<k_{t} \leqslant n$.

Proof. Since $\mathcal{C}=\left(m_{0}, \ldots, m_{N-1}\right)$ is an Eulerian cycle all elements of $V=\mathbb{F}_{q}^{k}$ appear exactly $q^{k}$ (corresponding to the size $|S|=q^{k}$ of the generating set $S$ ) times in $\mathcal{C}$. Therefore, the column vector $G m$ appears exactly $q^{k}$ times in $M$ for all $m$ $\in \mathbb{F}_{q}^{k}$. It now follows from Theorem 4 that $M$ is an orthogonal array; its multiplicity is just $q^{k}$ times the multiplicity of an OA constructed as in Theorem 4.

Let $m$ be an arbitrary element of $V=\mathbb{F}_{q}^{k}$ and $I_{m}:=\left\{j \mid m_{j}\right.$ $=m\}$. Then every element of $S=\mathbb{F}_{q}^{k}$ appears exactly once in the list $\left(m_{j+1}-m_{j} \mid j \in I_{m}\right)$ because $\mathcal{C}$ is an Eulerian cycle in $\Gamma(V, S)$ with multiplicity one (the addition $j+1$ is done modulo $N$ ). Consequently, the list of transitions that occur in $M$ from all columns of the form $G m$, i.e., $\left(G m_{j+1}-G m_{j} \mid j\right.$ $\in I_{m}$ ) is independent of $m$ and is equal (up to reordering the columns) to the orthogonal array $M^{\prime}:=\left(G e \mid e \in \mathbb{F}_{q}^{k}\right)$; it follows from Theorem 4 that $M^{\prime}$ is an orthogonal array. This proves that $M$ is Eulerian and also that $S_{k_{1}, \ldots, k_{t}}=G^{\times t}$ for all $t$ tuples since $M^{\prime}$ is an orthogonal array of strength $t$.

Note that our construction is closely related to Rötteler's construction [23]. In that paper Hamiltonian cycles in the Cayley graph $\Gamma\left(\mathbb{F}_{q}^{k}, S\right)$ are used, where the generating set is $S$ given by the $k$ coordinate vectors. The motivation behind this construction is to reduce the number of different control pulses in bang-bang decoupling.

Let us now explain how to construct decoupling schemes for general $t$-body Hamiltonians acting on $n$ qudits with bounded controls based on Theorem 5. To obtain a decoupling scheme using a minimal number of pulses we have to find a code $[n, k]_{q}$ such that $k$ is minimal and the dual distance $d^{\perp}$ is at least $t+1$. This may be formulated in terms of the dual code which has parameters $C^{\perp}=\left[n, n-k, d^{\perp}\right]_{q}$. The dual code $C^{\perp}$ should contain the maximally possible number of code words for given $n$ and $d^{\perp}$. This question is one of the central optimization problems in the theory of error correcting codes. To find such optimal or best known codes one could e.g. use the computer algebra system MAGMA [24] that contains a table of best linear codes known (i.e., with the maximal number of code words) for given length and minimal distance.

We now consider a quantum system consisting of $n$ qubits which are governed by a pair interaction Hamiltonian. For such a system we can construct decoupling schemes using $N$ pulses from an orthogonal array $\mathrm{OA}(N, n, 4,2)$. Hence, in order to apply Theorem 4 and Theorem 5 we have to find a linear code $C$ over $\mathbb{F}_{4}$ for which the parameters are $[n, k, d]$ and for which the dual distance is at least 3 . This can be done with the help of Hamming codes [22,23]. For every $m \in \mathbb{N}$ there is an orthogonal array $\mathrm{OA}\left[4^{m},\left(4^{m}-1\right) / 3,4,2\right]$. The columns of this OA are codewords of the dual code of a Hamming code. The corresponding Eulerian orthogonal array has parameters $\mathrm{OA}\left[16^{m},\left(4^{m}-1\right) / 3,4,2\right]$.

To obtain a decoupling scheme for a quantum system consisting of $n$ qubits, where $n$ is an arbitrary natural number, i.e., not necessarily of the form $n=\left(4^{m}-1\right) / 3$ we proceed as follows: first let $m \in \mathbb{N}$ be the unique integer such that $n \leqslant \frac{4^{m}-1}{3} \leqslant 4 n$. Then construct the orthogonal array with parameters $\mathrm{OA}\left[4^{m},\left(4^{m}-1\right) / 3,4,2\right]$ for bang-bang controls and the Eulerian orthogonal array with parameters $\mathrm{OA}\left[16^{m},\left(4^{m}-1\right) / 3,4,2\right]$ for bounded-strength controls, respectively. These results shows that the complexity of decoupling for general pair interaction Hamiltonians acting on $n$ qubits scales at most linearly in $n$ for bang-bang controls and at most quadratically in $n$ for bounded-strength controls, respectively.

\section{CONCLUSIONS AND DISCUSSIONS}

We have shown that it is possible to construct decoupling schemes using bounded-strength controls for composite multipartite qudit systems with the help of Eulerian orthogonal arrays. Our concept of Eulerian orthogonal arrays merges the 
desirable properties of usual orthogonal arrays (that were used to construct efficient decoupling schemes with bangbang controls) and Eulerian cycles that are at the heart of Viola and Knill's Eulerian decoupling method. We have shown how to construct efficient Eulerian orthogonal arrays based on linear error correcting codes. It would be interesting to find new constructions of Eulerian orthogonal arrays that yield decoupling schemes with a smaller number of pulses.

\section{ACKNOWLEDGMENTS}

The author would like to thank Anja Groch and Markus Grassl for helpful discussions. This work has been supported by the National Science Foundation under Grant No. EIA0086038 through the Institute for Quantum Information at the California Institute of Technology and the BMBF Project No. 01/BB01B.
[1] L. Viola, E. Knill, and S. Lloyd, Phys. Rev. Lett. 82, 2417 (1999).

[2] L. Viola and S. Lloyd, Phys. Rev. A 58, 2733 (1998).

[3] L. Viola, S. Lloyd, and E. Knill, Phys. Rev. Lett. 83, 4888 (1999).

[4] P. Zanardi, Phys. Lett. A 258, 77 (1999).

[5] J. A. Jones and E. Knill, J. Magn. Reson. 141, 323 (1999).

[6] D. Leung, J. Mod. Opt. 49, 1199 (2002).

[7] D. W. Leung, I. L. Chuang, F. Yamaguchi, and Y. Yamamoto, Phys. Rev. A 61, 042310 (2000).

[8] M. Stollsteimer and G. Mahler, Phys. Rev. A 64, 052301 (2001).

[9] P. Wocjan, M. Rötteler, D. Janzing, and T. Beth, Phys. Rev. A 65, 042309 (2002).

[10] C. H. Bennett, J. I. Cirac, M. S. Leifer, D. W. Leung, N. Linden, S. Popescu, and G. Vidal, Phys. Rev. A 66, 012305 (2002).

[11] J. L. Dodd, M. A. Nielsen, M. J. Bremner, and R. T. Thew, Phys. Rev. A 65, 040301(R) (2002).

[12] P. Wocjan, D. Janzing, and T. Beth, Quantum Inf. Comput. 2, 117 (2002).

[13] R. R. Ernst, G. Bodenhausen, and A. Wokaun, Principles of
Nuclear Magnetic Resonance in One and Two Dimensions (Clarendon Press, Oxford, 1987).

[14] J. S. Waugh, L. M. Huber, and U. Haeberlen, Phys. Rev. Lett. 20, 180 (1968).

[15] L. Viola, e-print quant-ph/0404038 (2004).

[16] L. Viola and E. Knill, Phys. Rev. Lett. 90, 037901 (2002).

[17] B. Bollobás, Modern Graph Theory, Graduate Texts in Mathematics Vol. 184 (Springer, New York, 1998).

[18] C. Godsil and G. Royle, Algebraic Graph Theory, Graduate Texts in Mathematics Vol. 207 (Springer, New York, 2001).

[19] Th. Beth, D. Jungnickel, and H. Lenz, Design Theory, Encyclopedia of Mathematics and Its Applications Vol. I, 2nd ed. (Cambridge University Press, Cambridge, 1999).

[20] The CRC Handbook of Combinatorial Designs, edited by C. J. Colbourn and J. H. Dinitz (CRC Press, Boca Raton, FL, 1996).

[21] A. S. Hedayat, N. J. A. Sloane, and J. Stufken, Orthogonal Arrays, Springer Series in Statistics (Springer, New York, 1999).

[22] M. Röttler and P. Wocjan, e-print quant-ph/0409135 (2004).

[23] M. Röttler, e-print quant-ph/0408078 (2004).

[24] W. Bosma, J. Cannon, and C. Playoust, J. Symb. Comput. 24, 235 (1997). 